\title{
Measure space on Weak Structure
}

\author{
Mohammed M. Khalaf*, Ahmed Elmoasry* \\ Department of Mathematics, Faculty of Science, Al-Azahar University, Asuiat, Egypt \\ Department of Mathematics, Aswan Faculty of Science, Aswan University, Aswan, Egypt
}

Abstract: Császár in [4] introduce a weak structure as generalization of general topology. The aim of this paper is to give basic concepts of the measure theory in weak structure.

Keywords: weak structure, $\sigma$-algebra, $\sigma$-additive function, Measures

\section{Notation and Preliminaries}

In mathematical analysis. Measurement theory plays a vital role in the expression completely for some mathematical concepts. In our research, we introduced some of the concepts of measurement in a weak structure. And we study their properties and some applications it. So we shall denote by $X$ nonempty set, by $\omega$ a weak structure [1] and by $P(X)$ the set of all parts (i.e., subsets) of $X$, and by $\phi$ the empty set. For any subset $\lambda$ of $X$ we shall denote by $\lambda^{c}$ its complements, i.e., $\lambda^{c}=\{x \in X \mid x \notin \lambda\}$. For any $\lambda, \mu \in P(X)$ we set $\lambda \mid \mu=$ $\lambda \cap \mu^{c}$. Let $\left(\lambda_{n}\right)$ be a sequence in $P(X)$.

The following Demorgan identity holds $\left(\bigcup_{n=1}^{\infty} \lambda_{n}\right)=\bigcap_{n=1}^{\infty} \lambda_{n}^{c}$. we define $\lim _{n \rightarrow \infty}\left(\vee \lambda_{n}\right)=\bigcap_{n=1}^{\infty}\left(\bigcup_{m=n}^{\infty} \lambda_{m}\right), \lim _{n \rightarrow \infty}\left(\wedge \lambda_{n}\right)=\bigcup_{n=1}^{\infty}\left(\bigcap_{m=n}^{\infty} \lambda_{m}\right)$. If $L=\lim \left(\underset{n \rightarrow \infty}{\vee} \lambda_{n}\right)=\lim \left(\bigwedge_{n \rightarrow \infty} \lambda_{n}\right)$, then we set $L=\lim _{n \rightarrow \infty}\left(\lambda_{n}\right)$, and we say that $\left(\lambda_{n}\right)$ converges to $L$. As easily checked, $\lim \left(\underset{n \rightarrow \infty}{\vee} \lambda_{n}\right)$ (resp., $\lim \left(\underset{n \rightarrow \infty}{\wedge} \lambda_{n}\right)$ consists of those elements of $X$ that belong to infinte elements of $\left(\lambda_{n}\right)$ ( resp., that belong to infinte elements of $\left(\lambda_{n}\right)$ expect perhaps a finite number. Therefore, $\lim \left(\bigwedge_{n \rightarrow \infty} \lambda_{n}\right) \subset \lim \left(\underset{n \rightarrow \infty}{\vee} \lambda_{n}\right)$. And it easy also to check that, if $\left(\lambda_{n}\right)$ is increasing $\left(\lambda_{n} \subset \lambda_{n+1}, n \in N\right)$, then $\quad \lim _{n \rightarrow \infty} \lambda_{n}=\bigcup_{n=1}^{\infty} \lambda_{n} \quad$ where, if $\quad\left(\lambda_{n}\right) \quad$ is decreasing $\quad\left(\lambda_{n} \supset \lambda_{n+1}, n \in N\right)$, then $\lim _{n \rightarrow \infty} \lambda_{n}=\bigcap_{m=n}^{\infty} \lambda_{n}$. In the first case we shall write $\lambda_{n} \uparrow L$, and in the second $\lambda_{n} \downarrow L$.

\section{Algebra and $\sigma$-algebra on a weake structure $\omega$}

Let $A$ be a nonempty subset of $\omega$

Definition 1.1 $A$ is said to be an algebra in $\omega$ if

a) $\phi \in \mathrm{A}$

b) $\lambda, \mu \in \mathrm{A} \Rightarrow \lambda \cup \mu \in \mathrm{A}$

c) $\lambda \in \mathrm{A} \Rightarrow \lambda^{c} \in \mathrm{A}$

Remark 1.1 It easy to see that, if $A$ is an algebra and $\lambda, \mu \in A$, then $\lambda \cap \mu$ and $\lambda \mid \mu$ belong to $A$. Therfore, the symmetric difference $\lambda \Delta \mu=(\lambda \mid \mu) \cup(\mu \mid \lambda)$ also belong to $\mathrm{A}$. Moreover, $\mathrm{A}$ is stable under finite union and intersection, that is $\lambda_{1}, \ldots \ldots \ldots, \lambda_{n} \in \mathrm{A} \Rightarrow\left\{\begin{array}{l}\lambda_{1} \cup \ldots \ldots \ldots \cup \lambda_{n} \in \mathrm{A} \\ \lambda_{1} \cap \ldots \ldots \ldots \cap \lambda_{n} \in \mathrm{A}\end{array}\right.$. 
Definition 1.2 An algebra $A$ in $\omega$ is said to be a $\sigma$-algebra if, for any sequence $\left(\lambda_{n}\right)$ of elements of $A$, we have $\bigcup_{n=1}^{\infty} \lambda_{n} \in \mathrm{A}$. We note that, if $\mathrm{A}$ is $\sigma$ - algebra and $\left(\lambda_{n}\right) \subset \mathrm{A}$, then $\bigcap_{n=1}^{\infty} \lambda_{n} \in \mathrm{A}$ owing to the De morgan identity.

Moreover, $\lim _{n \rightarrow \infty}\left(\wedge \lambda_{n}\right) \in A, \lim _{n \rightarrow \infty}\left(\vee \lambda_{n}\right) \in A$.

The following examples explain the difference between algebras and $\sigma$-algebras.

Example 1.1 Obviously, $P(X)$ and $\varepsilon=\{\phi\}$ are $\sigma$ - algebras in $X$. Morover, $\omega$ is the largest $\sigma$ algebras in $X$, and $\varepsilon$ is the smallest.

Example 1.2 In $[0,1)$, the class $\rho$ consisting of $\phi$, and of all finite unions

$\beta=\bigcup_{i=1}^{n}\left[a_{i}, b_{i}\right)$ with $0 \leq a_{i} \leq b_{i} \leq a_{i+1} \leq 1$ is an algebra.

Example 1.3 In an infinite set $X$ consider the class $\rho=\left\{\theta \in \omega \mid \theta\right.$ is finite, or $\theta^{c}$ is finite $\}$. Then $\rho$ is an algebra.

Example 1.4 In an uncountable set $X$ consider the class $\rho=\left\{\theta \in \omega \mid \theta\right.$ iscountable, or $\theta^{c}$ iscountable $\}$. Then $\rho$ is a $\sigma$ - algebra.

Definition 1.3 The intersection of all $\sigma$-algebras including $\tau \subseteq \omega$ is called the $\sigma$ - algebra generated by $\tau$, and will be denoted by $\sigma(\tau)$.

Example 1.5 Let $E$ be a metric space. The $\sigma$-algebra generated by all open subsets of $E$ is called the Borel $\sigma$ - algebra of $E$, and denoted by $B(E)$.

2. Measure

\subsection{Additive and $\sigma$ - additive functions}

Let $A \subset \omega$ be an algebra

Definition 2.1 Let $\mathrm{F}: \mathrm{A} \rightarrow 0,+\infty$ ] be such that $\mu(\phi)=0$.

(1) We say that $\mathrm{F}$ is additive if, for any family $A_{1}, \ldots . ., A_{n} \in \mathrm{A}$ of mutually disjoint sets, we have $\mathrm{F}$ ( $\left.\bigcup_{k=1}^{n} A_{k}\right)=\sum_{k=1}^{n} \mathrm{~F}\left(A_{k}\right)$.

(2) We say that $\mathrm{F}$ is $\sigma$-additive if, for any sequence $\left(A_{n}\right) \in \mathrm{A}$ of mutually disjoint sets such that $\bigcup_{k=1}^{\infty} A_{k} \in \mathrm{A}$, we have $\mathrm{F}\left(\bigcup_{k=1}^{\infty} A_{k}\right)=\sum_{k=1}^{\infty} \mathrm{F}\left(A_{k}\right)$.

Remarke 2.1 Let $A \subset \omega$ be an algebra

(1) Any $\sigma$-additive function on $A$ is also additive.

(2) If $\mathrm{F}$ is additive, $\lambda, \mu \in \mathrm{A}$, and $\lambda \supset \mu$, then $\mathrm{F}(\lambda)=\mathrm{F}(\mu)+\mathrm{F}(\lambda \mid \mu)$.

Therefore, $\mathrm{F}(\lambda) \geq \mathrm{F}(\mu)$. 
(3) Let $\mathrm{F}$ is additive on $\mathrm{A}$, and let $\left(A_{n}\right) \in \mathrm{A}$ be mutually disjoint sets such that $\bigcup_{k=1}^{\infty} A_{k} \in \mathrm{A}$. Then, $\mathrm{F}($ $\left.\bigcup_{k=1}^{\infty} A_{k}\right) \geq \sum_{k=1}^{n} \mathrm{~F}\left(A_{k}\right) \quad$ for all $\mathrm{n} \in \mathrm{N}$.

Therefore, $\quad \mathrm{F}\left(\bigcup_{k=1}^{\infty} A_{k}\right) \geq \sum_{k=1}^{\infty} \mathrm{F}\left(A_{k}\right)$

(4) Any $\sigma$-additive function $\mathrm{F}$ on $\mathrm{A}$ is also countably subadditive, that is, for any sequence $\left(A_{n}\right) \subset \mathrm{A}$ such that $\bigcup_{k=1}^{\infty} A_{k} \in \mathrm{A}, \quad \mathrm{F}\left(\bigcup_{k=1}^{\infty} A_{k}\right) \leq \sum_{k=1}^{\infty} \mathrm{F}\left(A_{k}\right)$.

(5) Inview of parts 3 and 4 an additive function is $\sigma$-additive if and only if it is countably subadditive.

Definition 2.2 A $\sigma$-additive function $F$ on an algebra $A \subset \omega$ is said to be

(1) finite if $\mathrm{F}(X)<\infty$,

(2) $\sigma$ - finite if there exists a sequence sequence $\left(A_{n}\right) \subset \mathrm{A}$

such that $\bigcup_{n=1}^{\infty} A_{n}=X$, and $\mathrm{F}\left(A_{n}\right)<\infty$ for all $\mathrm{n} \in \mathrm{N}$.

Example 2.1 In $X=N$, consider the algebra $\mathrm{A}=\left\{A \in \omega \mid\right.$ Aisfinite, or $A^{c}$ finite $\}$. The function $\mathrm{F}: \mathrm{A} \rightarrow 0, \infty]$ defined as $\mathrm{F}(A)=\left\{\begin{array}{ccc}n(A) & \text { if } A \text { finite } \\ \infty & \text { if } A^{c} \text { finite }\end{array}\right.$ (where $n(A)$ ) stands for the number of elements of $A$ is $\sigma$-additive. On the other hand.

The function $\mathrm{F}: \mathrm{A} \rightarrow 0, \infty]$ defined as $\mathrm{F}(A)=\left\{\begin{array}{ccc}\sum_{n \in A} \frac{1}{2^{n}} & \text { if } & A \text { finite } \\ \infty & \text { if } & A^{c} \text { finite }\end{array}\right.$ is additive but not $\sigma-$ additive.

Theorem 2.1 Let $\mu$ be additive on $\mathrm{A}$. Then $(i) \Leftrightarrow(i i)$ where:

(i) $\mu$ is $\sigma$-additive,

(ii) $\left(A_{n}\right)$ and $A \subset \mathrm{A}, A_{n} \uparrow A \Rightarrow \mu\left(A_{n}\right) \uparrow \mu(A)$.

Proof $(i) \Rightarrow(i i)$ Let $\left(A_{n}\right), A \subset \mathrm{A}, A_{n} \uparrow A$. Then, $A=A_{1} \cup \bigcup_{n=1}^{\infty}\left(A_{n+1} \backslash A_{n}\right)$, the a bove being disjoint union. Since $\mu$ is $\sigma-$ additive, we deduce that $\mu(A)=\mu\left(A_{1}\right)+\sum_{n=1}^{\infty}\left(\mu\left(A_{n+1}\right)-\mu\left(A_{n}\right)\right)=\lim _{n \rightarrow \infty} \mu\left(A_{n}\right)$, and (ii) follows.

(ii) $\Rightarrow($ i $)$ Let $\left(A_{n}\right) \subset \mathrm{A}$ be a sequence of mutually disjoint sets such that $A=\bigcup_{k=1}^{\infty} A_{k} \in \mathrm{A}$. Define $B_{n}=\bigcup_{k=1}^{\infty} A_{k}$. Then $B_{n} \uparrow A$. So, in view of $(i i), \mu\left(B_{n}\right)=\sum_{n=1}^{\infty} \mu\left(A_{k}\right) \uparrow \mu\left(A_{n}\right)$.

This implies $(i)$ 
Definition 2.2 let $\varepsilon=\{\phi\}$ are $\sigma$-algebras in $X$.

(1) We say that the pair $(X, \varepsilon)$ is a measurable space.

(2) A $\sigma$-additive function $\mu: \varepsilon \rightarrow 0,+\infty]$ is called a measure on $(X, \varepsilon)$

(3) The triple $(X, \varepsilon, \mu)$, where $\mu$ is a measure on a measurable space $(X, \varepsilon)$ is called a measurable space

(4) A measure $\mu$ is said to be complete if $A \in \varepsilon, B \subset A, \mu(A)=0 \Rightarrow$

$B \in \varepsilon($ and so $\mu(B)=0)$.

(5) A measure $\mu$ is said to be concentrated on a set $A \in \varepsilon$ if $\mu\left(A^{c}\right)=0$.

In this case we say that $A$ is a support of $\mu$

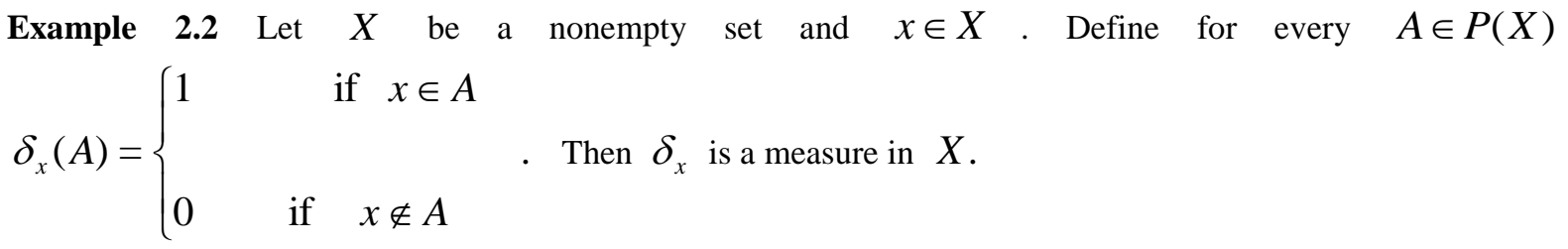

\section{References}

[1] A. Császár, Generalized open sets, Acta Math. Hungar 75 (1997), 65-87.

[2] A. Császár, Generalized topology, generalized continuity, Acta Math. Hungar 96 (2002), 351-357.

[3] A. Császár, Generalized open sets in generalized topologies, Acta Math. Hungar 106 (2005), 53-66.

[4] A. Császár, Weak structures, Acta Math. Hungar 131(1-2) (2011), 193-195.

[5] J. Umehara H. Maki and T Noiri, Every topological space is pret1/2, Mem. Fac. Sci.Kochi.Univ. Ser. A Math. 17 (1996), $33-42$. 